\title{
List of Participants of the Lipmann Symposium
}

ACS, G.

The Mt. Sinai School of Medicine, New York, USA

AUHAGEN, E.

Treasurer of the Gesellschaft für Biologische Chemie,

Wuppertal, Germany

BADDILEY, J.

The University of Newcastle, Newcastle upon Tyne, England

BAUER, K.

Technische Universität Berlin, Berlin, Germany

BENNETT, T.P.

Florida State University, Tallahasse, USA

BERGMEYER, H.-U.

Boehringer Mannheim Company, Tutzing, Germany

BESSMAN, S. P.

University of Southern California, Los Angeles, USA

BLOEMENDAL, $\mathrm{H}$.

University of Nijmegen, Nijmegen, The Netherlands

BOMAN, H. G.

University of Umea, Umea, Sweden

BRODIE, A. F.

University of Southern California, Los Angeles, USA

CHANTRENNE, $\mathrm{H}$.

Laboratoire de Chimie Biologique, Bruxelles, Belgium

CHAPEVILLE, F.

Institut de Biologie Moléculaire, Paris, France

CRAMER, F.

Max-Planck-Institut für Experimentelle Medizin, Göttingen,

Germany

CROKAERT, R.

Université Libre de Bruxelles, Bruxelles, Belgium

EBASHI, $S$.

University of Tokyo, Tokyo, Japan 
EHRENSTEIN, G. von

Max-Planck-Institut für Experimentelle Medizin, Göttingen,

Germany

EIGEN, M.

Max-Planck-Institut für Biophysikalische Chemie, Göttingen,

Germany

ELLIOTT, W. H.

University of Adelaide, Adelaide, Australia

ENGSTRÖM, L.

Institute of Medical Chemistry, Uppsala, Sweden

FISCHER, $H$.

Max-Planck-Institut für Immunbiologie, Freiburg, Germany

FOX, J. L.

University of Texas, Austin, USA

GERLACH, $u$.

Universität Münster, Münster, Germany

GIBIAN, $H$.

Schering AG, Berlin, Germany

GOLDBERG, I. H.

Harvard Medical School, Boston, USA

GORDON, J.

Friedrich Miescher Institut, Basel, Switzerland

HAENNI, A. L.

Institut de Biologie Moléculaire, Paris, France

HARTMANN, G.

Universität München, München, Germany

HESS, B.

Max-Planck-Institut für Ernährungsphysiologie, Dortmund,

Germany

HELMREICH, E.

Chairman of the Gesellschaft für Biologische Chemie,

Würzburg, Germany

HERRLICH, P.

Max-Planck-Institut für Molekulare Genetik, Berlin, Germany

HIEROWSKI, M.

University of Aarhus, Aarhus, Denmark 
HILDEBRAND, J. G.

Harvard Medical School, Boston, USA

HUBERMAN, A.

Instituto Nacional de la Nutrición, Mexico City, Mexico

HÜLSMANN, W.C.

Erasmus University, Rotterdam, The Netherlands

JONES, M. E.

University of Southern California, Los Angeles, USA

KLEINKAUF, $\mathrm{H}$.

Technische Universität Berlin, Berlin, Germany

KREBS, H. A.

Oxford, England

KRISKO, I.

Veterans Administration Hospital, Houston, USA

KUCAN, Z .

"Rugjer Bošković" Institute, Zagreb, Yugoslavia

LIPMANN, F.

The Rockefeller University, New York, USA

LYNEN, F.

Max-Planck-Institut für Biochemie, Martinsried/München,

Germany

MANO, Y.

University of Tokyo, Tokyo, Japan

MARCHIS-MOUREN, G.

Institut de Chimie Biologique, Marseille, France

M $\varnothing$ LLER, K.

Carlsberg Laboratorium, Copenhagen, Denmark

NIEMEYER, H.

Universidad de Chile, Santiago, Chile

NOSE, $Y$.

Kyoto Prefectural University of Medicine, Kyoto, Japan

OFENGAND, J.

Roche Institute of Molecular Biology, Nutley, USA

ORREGO, F.

Instituto Nacional de Cardiologia, Mexico City, Mexico

PARK, J.H.

Vanderbilt University Medical School, Nashville, USA 
PARMEGGIANI, A.

Gesellschaft für Molekularbiologische Forschung,

Stöckheim/Braunschweig, Germany

RICHTER, D.

Universität Hamburg, Hamburg, Germany

ROSKOSKI, R.

University of lowa, lowa City, USA

SCHUSTER, H.

Max-Planck-Institut für Molekulare Genetik, Berlin, Germany SCHWEIGER, M.

Max-Planck-Institut für Molekulare Genetik, Berlin, Germany SPECTOR, L.

The Rockefeller University, New York, USA

SRERE, P.A.

The University of Texas, Dallas, USA

STADTMAN, E.R.

National Institutes of Health, Bethesda, USA

SY, J .

The Rockefeller University, New York, USA

SZAFRANSKI, P.

Polish Academy of Sciences, Warsaw, Poland

TAKEDA, M.

Kobe University, Kobe, Japan

TAO, M.

University of Illinois, Chicago, USA

TRAUT, R. R.

University of California, Davis, USA

TRAUTNER, T. A.

Max-Planck-Institut für Molekulare Genetik, Berlin, Germany

URETA, T.

Universidad de Chile, Santiago, Chile

VENNESLAND, B.

Forschungsstelle Vennesland, Berlin, Germany

WAKABAYASHI, $K$.

University of Tokyo, Tokyo, Japan 


\section{XVIII}

WITT, H. T.

Technische Universität Berlin, Berlin, Germany

WITTMANN, H. G.

Max-Planck-Institut für Molekulare Genetik, Berlin, Germany WITTMANN-LIEBOLD, B .

Max-Planck-Institut für Molekulare Genetik, Berlin, Germany ZACHAU, H. G.

Universität München, München, Germany 